\title{
Erratum: Study of the Mass and Spin-Parity of the Higgs Boson Candidate via Its Decays to $Z$ Boson Pairs [Phys. Rev. Lett. 110, 081803 (2013)]
}

\author{
S. Chatrchyan et al. * \\ (CMS Collaboration) \\ (Received 10 April 2013; published 2 May 2013)
}

DOI: 10.1103/PhysRevLett.110.189901

PACS numbers: 14.80.Bn, 12.60.-i, 13.85.Qk, 13.88.+e, 99.10.Cd

During a review of our Letter, we found an error in the sentence "Under the assumption of spin 0, the test statistic formed from a profile likelihood ratio $\lambda=\mathcal{L}_{0^{-}} / \mathcal{L}_{0^{+}}$of the $0^{-}$and $0^{+}$hypotheses yields a $p$-value of $0.072 \%$ for $0^{-}$and a $p$-value of 0.7 for $0^{+}$, with $-2 \ln \lambda=5.5$ favoring $0^{+}$."

The $p$-value for $0^{-}$should be replaced with $0.72 \%$, resulting in "Under the assumption of spin 0 , the test statistic formed from a profile likelihood ratio $\lambda=\mathcal{L}_{0^{-}} / \mathcal{L}_{0^{+}}$of the $0^{-}$and $0^{+}$hypotheses yields a $p$-value of $0.72 \%$ for $0^{-}$and a $p$-value of 0.7 for $0^{+}$, with $-2 \ln \lambda=5.5$ favoring $0^{+} . "$

\footnotetext{
*Full author list given at the end of the Letter.
}

Published by the American Physical Society under the terms of the Creative Commons Attribution 3.0 License. Further distribution of this work must maintain attribution to the author(s) and the published article's title, journal citation, and DOI. 